\title{
Myeloid Neoplasms with Germline Predisposition without a Preexisting Disorder or Organ Dysfunction
}

National Cancer Institute

\section{Source}

National Cancer Institute. Myeloid Neoplasms with Germline Predisposition without a

Preexisting Disorder or Organ Dysfunction. NCI Thesaurus. Code C151897.

Familial myeloid neoplasms associated with germline mutations without a preexisting disorder or organ dysfunction. 